\title{
Association between high fatty liver index and development of colorectal cancer: a nationwide cohort study with 21,592,374 Korean
}

\author{
Yoon Jin $\mathrm{Choi}^{1,2}$, Dong Ho Lee ${ }^{1,3}$, and Kyung-Do Han ${ }^{4}$
}

\begin{abstract}
${ }^{1}$ Department of Internal Medicine, Seoul National University Bundang Hospital, Seongnam; ${ }^{2}$ Department of Internal Medicine, Korea University Guro Hospital, Seoul; ${ }^{3}$ Department of Internal Medicine and Liver Research Institute, Seoul National University College of Medicine, Seoul; ${ }^{4}$ Department of Biostatistics, College of Medicine, The Catholic University of Korea, Seoul, Korea
\end{abstract}

Received: January 15, 2018 Revised : April 26, 2018 Accepted: December 19, 2018

\author{
Correspondence to \\ Dong Ho Lee, M.D. \\ Department of Internal \\ Medicine, Seoul National \\ University Bundang Hospital, \\ 82 Gumi-ro 173beon-gil, \\ Bundang-gu, Seongnam 13620, \\ Korea \\ Tel: $+82-31-787-7008$ \\ Fax: +82-31-787-4051 \\ E-mail:dhljohn@yahoo.com
}

\begin{abstract}
Background/Aims: In Korea, the incidence of colorectal cancer (CRC) and nonalcoholic fatty liver disease (NAFLD) has increased due to a westernized lifestyle. This study investigated whether a high fatty liver index that reflects NAFLD correlates with CRC.

Methods: Data from the National Health Insurance Corporation 2009 to 2012 were analyzed. NAFLD disease was defined as a fatty liver index $>60$ in the absence of alcohol consumption of $\geq 30 \mathrm{~g} /$ day.

Results: NAFLD was identified in 2,543,649 (11.8\%) of 21,592,374 participants. CRC was identified in 19,785 ( $0.8 \%$ ) of participants with NAFLD (fatty liver index $\geq 60$ ) and in 80,871 (0.6\%) participants without NAFLD (fatty liver index <30). Multivariate logistic regression analysis demonstrated an independent association between NAFLD and CRC after adjusting for other confounders (hazard ratio, 1.13; odds ratio, 1.12 to 1.15). In subgroup analyses, fatty liver index $\geq 60$ was associated with CRC regardless of body mass index, but the association was more prominent in persons with a normal index. NAFLD, in the absence of diabetes, hypertension, or dyslipidemia, was more highly associated with CRC than when one or more of these conditions are present.

Conclusions: CRC should be considered as a possibility in patients with fatty liver index $\geq 60$, even in the absence of obesity or other metabolic syndromes.
\end{abstract}

Keywords: Colorectal neoplasms; Non-alcoholic fatty liver disease; Metabolic syndrome

\section{INTRODUCTION}

Colorectal cancer (CRC) is one of the most prevalent cancers in men and women. Globally, 1.2 million new cases of CRC and 608,700 CRC-related deaths were estimated to have occurred in $2008[1,2]$. The prevalence of cancers of the colon and rectum appears to have increased among younger adults. Indeed, Korea has one of the highest rates of growth in the prevalence of CRC [3,4] exceeding the incidence noted in the United States. An increase of CRC is attributable to obesity and metabolic syndrome associated with a now prevalent westernized lifestyle [5]. Several studies suggest that obesity and metabolic syndrome are risk factors for CRC [6,7].

Nonalcoholic fatty liver disease (NAFLD), one of the main liver diseases worldwide [8], has been considered the hepatic manifestation of metabolic syndrome [9]. Accumulating evidence supports the hypothesis that NAFLD precedes the development of obesity or diabetes and is not solely a hepatic manifestation [10]. NAFLD 
independently predicts insulin resistance, enabling physicians to identify certain individuals with insulin resistance who may be missed by metabolic syndrome criteria [10]. This finding suggests that NAFLD may be associated with development of CRC even before fullblown metabolic syndrome is observed. However, only limited studies have evaluated this relationship [11,12].

Fatty liver index (FLI) is calculated using body mass index (BMI), waist circumference, triglyceride (TG), and gamma $(\gamma)$-glutamyl transferase (GGT), and is used as a noninvasive means to predict NAFLD [13]. The formula is validated for high diagnostic accuracy in a large population $[14,15]$.

This study aimed to evaluate the association between NAFLD, as defined by FLI, and development of CRC using Korean nationwide data.

\section{METHODS}

\section{Data source and study population}

The database of the National Health Insurance Corporation (NHIC) was used. NHIC is a national insurer managed by the Korean government to which approximately $97 \%$ of the Korean population subscribes. The NHIC recommends that subscribers undergo a standardized medical examination at least biennially. Any researcher can use the NHIC database after the official review committee approves study protocols. Operational definitions from the International Classification of Diseases, 1oth revision codes were used to define disease groups.

Reports for 23,503,802 individuals who had undergone an annual or biennial evaluation between 2009 and 2012 were available in the NHIC database. Because of insufficient data, 146,156 participants were excluded, Further, 45,341 persons aged less than 20 years or older than 85 years; 45,341 individuals diagnosed with liver cirrhosis (K703), 361,641 with hepatitis (K746), and 14,955 with hepatic cancer (C22) were also excluded. Finally, 1,117,343 heavy alcohol consumers (daily alcohol consumption $\geq 30 \mathrm{~g}$ for men and $\geq 20 \mathrm{~g}$ for women), and 64,813 participants diagnosed with CRC before 2009 were also excluded to avoid enrolling patients with pre-existing diseases. The remaining 21,592,374 participants were analyzed and followed up. A summary of the study population selection is illustrated in Fig. 1. This study was

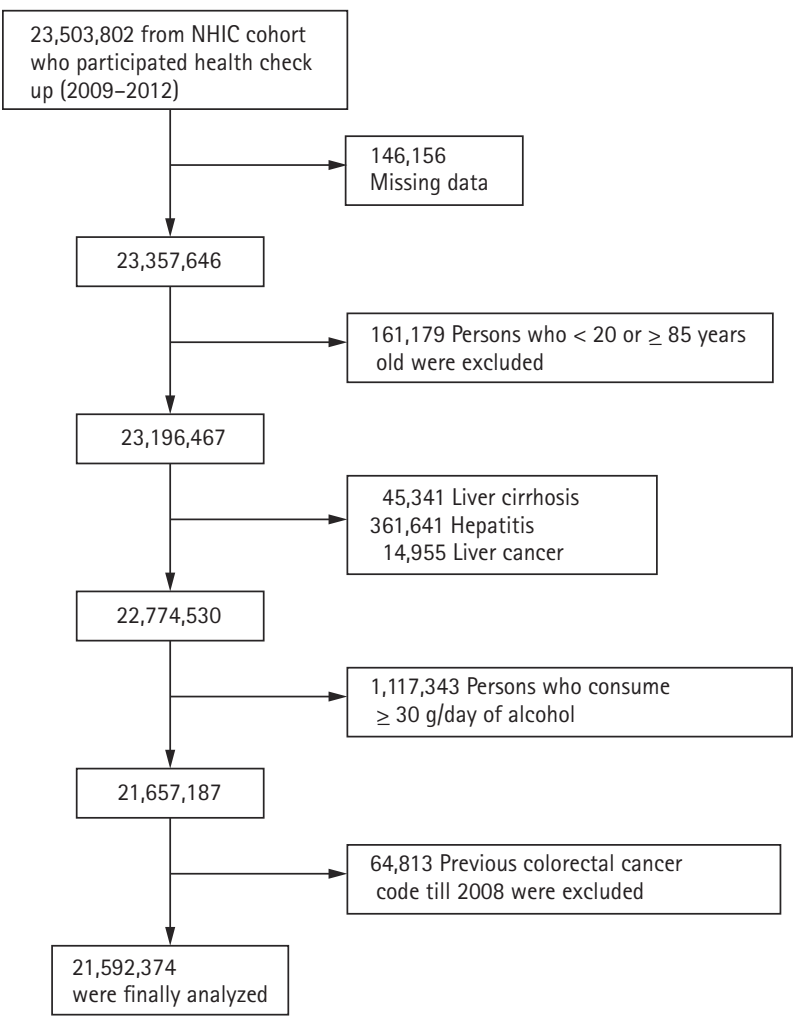

Figure 1. Flow chart showing the enrollment process for the study cohort. NHIC, National Health Insurance Corporation.

approved by the Institutional Review Board of Seoul National University Bundang Hospital (X-1608/360-904). All procedures performed in studies involving human participants were in accordance with the 1964 Helsinki declaration and its later amendments or comparable ethical standards. Since the present study involved routinely collected data, additional specific informed consent was not required.

\section{Measurements of clinical parameters and biochemical analysis}

Standardized self-reporting questionnaires were used to collect data at the time of enrollment for the following variables, which are regarded as risk factors for liver injury. These variables were included as covariates in multivariable analyses: age (years), sex (male or female), residency (rural and urban), yearly income (lower quintile vs. the remaining quintiles), alcohol consumption (frequency of alcohol consumption in 1 week and the amount of alcohol consumed on one occasion were evaluated by reviewing the questionnaire [frequency: o 
to 7 days/week and amount: drinks per occasion]), and cigarette smoking (never, former, and current). Regular exercise was defined as engaging in vigorous exercise on a regular basis (high-intensity activity $\geq 3$ times/ week or moderate-intensity activity $\geq 5$ times/week) [16]. $\mathrm{BMI}$ and systolic and diastolic blood pressures $(\mathrm{mmHg})$ were also measured. Participants were considered obese when their BMI was $\geq 25 \mathrm{~kg} / \mathrm{m}^{2}$ based on criteria from the Asian-Pacific region [17]. Waist circumference $>90$ cm was defined as abdominal obesity [17]. Diabetes mellitus (DM) was defined based on use of insulin or oral hypoglycemic agents [8] or a fasting plasma glucose level $\geq 126 \mathrm{mg} / \mathrm{dL}$ [18]. Participants were diagnosed as hypertensive if their systolic pressure was $\geq 140 \mathrm{mmHg}$, if their diastolic pressure was $\geq 90 \mathrm{mmHg}$, or if they currently used antihypertensive medication [19]. After overnight fasting for at least 8 hours, blood specimens collected from each participant were processed and transported in cold storage to the Central Testing Institute (Neodin Medical Institute, Seoul, Korea). Levels of total cholesterol (mg/dL) and liver enzymes alanine aminotransferase (ALT), aspartate aminotransferase (AST), and GGT (IU/L) in the serum were determined.

The primary endpoint for the study was newly diagnosed CRC, which was defined using the International Classification of Diseases, 10th revision codes (C180200). The diagnosis of CRC was considered new when a patient had no such diagnosis during the preceding years.

\section{Surrogate measure of fatty liver}

Screening of asymptomatic individuals using imaging modalities, such as ultrasonography (US) and computed tomography, are expensive for mass screening [20]. Therefore, simple, noninvasive tests such as the FLI were used. To identify fatty liver, FLI was calculated according to an algorithm based on TG levels, BMI, GGT, and waist circumference: $\mathrm{FLI}=\left(\mathrm{e}^{0.953 \times \mathrm{Ln}[\mathrm{TG}]+0.139 \times \mathrm{BMI}+}\right.$ $0.718 \times \operatorname{Ln}[\mathrm{GGT}]+0.053 \times$ waist circumference -15.745$) /\left(1+\mathrm{e}^{0.953 \times \operatorname{Ln}[\mathrm{TG}]}\right.$ $+0.139 \times \mathrm{BMI}+0.718 \times \operatorname{Ln}[\mathrm{GGT}]+0.053 \times$ waist circumference -15.745$) \times 100$.

FLI ranges from o to 100. It has been validated against fatty liver diagnosis in the US with a proven accuracy of 0.84 (95\% confidence interval [CI], o.81 to o.87). Participants were categorized into three FLI groups, $<30$, the probability of not having fatty liver is $91 \% ; 30 \leq \mathrm{FLI}<60$, an intermediate probability; and $\geq 60$, the probability of having fatty liver is $78 \%$ [21]. Participants were classified as having NAFLD if FLI was $\geq 60$ in the absence of other causes of chronic liver disease (e.g., a history of hepatitis or cirrhosis, hepatitis B surface antigen negativity, and excessive alcohol consumption [ $\geq 30 \mathrm{~g} /$ day]).

\section{Statistical analyses}

Data are presented as mean \pm standard deviation for normally distributed continuous variables and as proportions for categorical variables. Student $t$ tests and analysis of variance were used to analyze continuous variables, and differences between nominal variables were compared with chi-square tests. Incidence rates of cancers were calculated by dividing the number of events by person-time at risk. To evaluate the independent association of FLI with risk of cancer incidence, the Cox proportional hazard model was used after adjusting for age, smoking status, alcohol consumption, exercise, and BMI. Subgroup analyses were performed according to age, smoking status, alcohol consumption, the presence of DM, metabolic syndrome signs and symptoms, obesity, abdominal obesity, physical activity, and yearly income. Statistical analyses were performed using SAS version 9.4 (SAS Institute, Cary, NC, USA) and R version 3.2.3 (The R Foundation for Statistical Computing, Vienna, Austria). A 2-sided $p<0.05$ was considered statistically significant. The cutoff of $p$ for interaction was 0.15 .

\section{RESULTS}

\section{Demographic characteristics}

Participants were divided into three groups based on FLI; baseline characteristics of the study participants are provided in Table 1. Anthropometrics, clinical variables, and biochemistry data were analyzed according to FLI grouping. Among 21,592,374 enrollees, 2,543,649 persons (11.8\%) were defined as having NAFLD based on FLI.

Participants with NAFLD in the FLI $\geq 6$ group were predominantly male compared to the other two groups. Mean age of the three groups were different. The group characterized by $30 \leq \mathrm{FLI}<60$ was older than the other two groups. The FLI $\geq 60$ group included more individuals who smoked currently and consumed alcohol. Moreover, participants with an FLI $\geq 60$ had higher values for waist circumference, BMI, blood pressure, fast- 
Table 1. Demographic characteristics of the study subjects

\begin{tabular}{|c|c|c|c|c|}
\hline Variable & $\begin{array}{c}\text { FLI }<30 \\
(n=14,236,480)\end{array}$ & $\begin{array}{l}30 \leq \mathrm{FLI}<60 \\
(\mathrm{n}=4,812,245)\end{array}$ & $\begin{array}{c}\text { FLI } \geq 60 \\
(n=2,543,649)\end{array}$ & $p$ value $^{a}$ \\
\hline Male sex & $5,319,211(37.4)$ & $3,170,656(65.9)$ & $1,999,161(78.6)$ & $<0.001$ \\
\hline Age, yr & $46.3 \pm 14.5$ & $51.3 \pm 13.6$ & $48.5 \pm 12.9$ & $<0.001$ \\
\hline$<40$ & $4,617,518(32.4)$ & $995,453(20.6)$ & $667,250(26.2)$ & \\
\hline $40-60$ & $7,807,138(54.8)$ & $2,926,809(60.8)$ & $1,555,383(61.2)$ & \\
\hline$\geq 60$ & $1,811,824(12.7)$ & $889,983(18.5)$ & $321,016(12.6)$ & \\
\hline BMI, kg/m ${ }^{2}$ & $22.2 \pm 2.4$ & $25.6 \pm 2.3$ & $28.1 \pm 3.1$ & $<0.001$ \\
\hline $\mathrm{WC}, \mathrm{cm}$ & $75 \cdot 4 \pm 7 \cdot 1$ & $86.0 \pm 5 \cdot 5$ & $92.4 \pm 6.9$ & $<0.001$ \\
\hline $\mathrm{BMI} \geq 25 \mathrm{~kg} / \mathrm{m}^{2}$ & $1,788,564(12.6)$ & $2,841,852(59.1)$ & $2,184,201(85.9)$ & $<0.001$ \\
\hline $\mathrm{WC} \geq 90 \mathrm{~cm}$ & $2,031,463(14 \cdot 3)$ & $2,242,639(46.6)$ & $1,821,347(71.6)$ & $<0.001$ \\
\hline $\mathrm{SBP}, \mathrm{mmHg}$ & $118.9 \pm 14.5$ & $126.7 \pm 14.5$ & $130.0 \pm 14.6$ & $<0.001$ \\
\hline $\mathrm{DBP}, \mathrm{mmHg}$ & $73.9 \pm 9.6$ & $78.7 \pm 9.6$ & $81.4 \pm 9.9$ & $<0.001$ \\
\hline Serum glucose level, mg/dL & $93.7 \pm 18.5$ & $101.9 \pm 26.0$ & $108.2 \pm 32.3$ & $<0.001$ \\
\hline Serum cholesterol level, mg/dL & $189 \pm 34.7$ & $203.1 \pm 37.2$ & $211.3 \pm 39 \cdot 5$ & $<0.001$ \\
\hline Serum triglyceride level, mg/dL & $86.8(86.8-86.9)$ & $153.3(153.2-153 \cdot 4)$ & $226.4(226.3-226.6)$ & $<0.001$ \\
\hline ALT level, IU/L b & $18.1(18.1-18.1)$ & $26.9(26.87-26.9)$ & $37.7(37.7-37 \cdot 7)$ & $<0.001$ \\
\hline AST level, IU/L ${ }^{b}$ & $22.2(22.2-22.3)$ & $26.0(26.0-26.1)$ & $31.4(31.3-31.5)$ & $<0.001$ \\
\hline GGT level, IU/L & $19.4(19.4-19.4)$ & $36.3(36.3-36.4)$ & $64.5(64 \cdot 4-64.6)$ & $<0.001$ \\
\hline Current smoker & $2,505,512(17.6)$ & $1,397,788(29.1)$ & $1,001,952(39.4)$ & $<0.001$ \\
\hline Alcohol consumption $^{\mathrm{c}}$ & $5,519,637(38.8)$ & $2,343,068(48.7)$ & $1,537,409(60.4)$ & $<0.001$ \\
\hline Regular physical exercise $^{\mathrm{d}}$ & $2,485,371(17.5)$ & $894,077(18.6)$ & $426,799(16.8)$ & $<0.001$ \\
\hline Lower quintile of yearly income & $3,124,602(22.0)$ & $938,595(19 \cdot 5)$ & $491,132(19 \cdot 3)$ & $<0.001$ \\
\hline Diabetes & $777,405(5 \cdot 5)$ & $662,415(13.8)$ & $505,886(19.9)$ & $<0.001$ \\
\hline Hypertension & $2,588,109(18.2)$ & $1,806,013(37.5)$ & $1,169,468(46.0)$ & $<0.001$ \\
\hline Dyslipidemia $^{\mathrm{e}}$ & $1,917,058(13.5)$ & $1,330,990(27 \cdot 7)$ & $920,307(36.2)$ & $<0.001$ \\
\hline$\geq 1$ metabolic syndrome component ${ }^{f}$ & $3,993,359(28.1)$ & $2,593,342(53.9)$ & $1,666,824(65.5)$ & $<0.001$ \\
\hline Development of CRC & $80,871(0.6)$ & $40,612(0.8)$ & $19,785(0.8)$ & $<0.001$ \\
\hline Follow-up duration, yr & $5 \cdot 3 \pm 1.1$ & $5 \cdot 3 \pm 1.1$ & $5 \cdot 3 \pm 1.1$ & NS \\
\hline
\end{tabular}

Values are presented as number (\%) or mean $\pm \mathrm{SD}$.

FLI, fatty liver index; BMI, body mass index; WC, waist circumference; SBP, systolic blood pressure; DBP, diastolic blood pressure; ALT, alanine transaminase; AST, aspartate aminotransferase; GGT, gamma glutamyltransferase; CRC, colorectal cancer; NS, not significant.

${ }^{\mathrm{a} A m o n g}$ three groups.

${ }^{\mathrm{b}}$ Geometric mean (95\% confidence interval).

${ }^{\mathrm{c}}$ Persons who consumed alcohol $\geq 30 \mathrm{~g} /$ day were initially excluded.

${ }^{\mathrm{d}}$ Persons who did not perform high intensity activity $\geq 3$ times/week or moderate intensity activity $\geq 5$ times/week.

${ }^{\mathrm{e}}$ Dyslipidemia was defined as a triglyceride level $\geq 150 \mathrm{mg} / \mathrm{dL}$ or use of a lipid-lowering drug.

${ }^{\mathrm{f}}$ Metabolic syndrome components included hypertension, dyslipidemia, and diabetes mellitus.

ing glucose level, TG level, total cholesterol level, AST level, ALT level, and GGT level than those in the other two groups. When the total population was divided into two groups, FLI $<60$ and FLI $\geq 60$, the latter included more individuals who exercised less frequently and fewer individuals who were in the lowest quintile of yearly income (Supplementary Table 1).

Of 2,543,649 participants with NAFLD defined by the 
Table 2. Results of multivariable analyses of the impact of the FLI on the risk of colorectal cancer in the Korean population

\begin{tabular}{|c|c|c|c|c|c|c|}
\hline \multirow{2}{*}{ FLI } & \multirow{2}{*}{ Number } & \multirow{2}{*}{ Event } & \multirow{2}{*}{$\mathrm{IR}^{\mathrm{a}}$} & \multicolumn{3}{|c|}{$\mathrm{HR}(95 \% \mathrm{CI})$} \\
\hline & & & & Model ${ }^{b}$ & Model $2^{c}$ & $\operatorname{Model}_{3}{ }^{\mathrm{d}}$ \\
\hline $\mathrm{FLI}<30$ & $14,236,480$ & 80,871 & 1.07 & 1 (Reference) & 1 (Reference) & 1 (Reference) \\
\hline $30 \leq \mathrm{FLI}<60$ & $4,812,245$ & 40,612 & 1.58 & $1.09(1.08-1.11)$ & $1.09(1.08-1.10)$ & $1.07(1.06-1.09)$ \\
\hline $\mathrm{FLI} \geq 60$ & $2,543,649$ & 19,785 & 1.48 & $1.18(1.16-1.20)$ & $1.17(1.15-1.19)$ & $1.13(1.12-1.15)$ \\
\hline$p$ for trend & & & & $<0.001$ & $<0.001$ & $<0.001$ \\
\hline
\end{tabular}

FLI, fatty liver index; IR, incidence rate; HR, hazard ratios; CI, confidential interval.

${ }^{\mathrm{a}} 1,000$ person-year.

${ }^{\mathrm{b}}$ Data are adjusted for age, sex.

${ }^{\mathrm{c}}$ Data are adjusted for age, sex, smoking status, alcohol consumption, regular physical exercise and income.

${ }^{\mathrm{d}}$ Data are adjusted for age, sex, smoking status, alcohol consumption, regular physical exercise, income, diabetes mellitus, hypertension, and dyslipidemia.

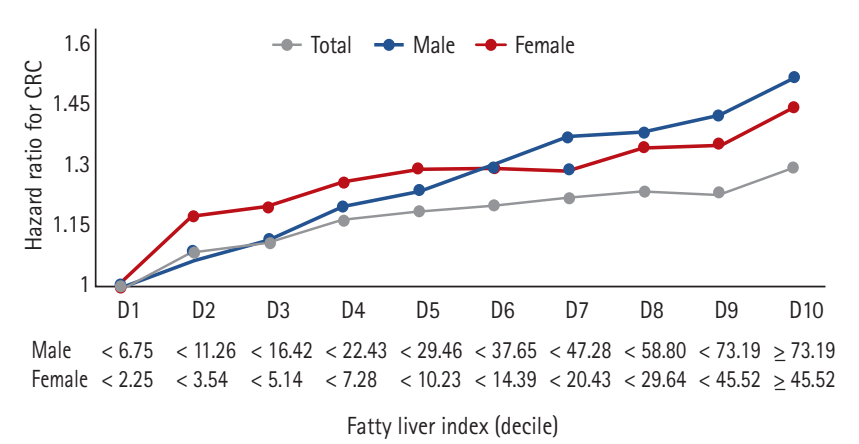

Figure 2. Hazard ratios of the development of colorectal cancer based on the fatty liver index. Data are adjusted for age, smoking status, alcohol consumption, regular exercise, and yearly income. CRC, colorectal cancer; D, decile.

FLI, 19,785 (0.78\%) developed CRC, while 80,871 (0.57\%) and 40,612 (o.84\%) were diagnosed as having CRC among 14,236,480 persons with an FLI < 30 and 4,812,245 individuals with $30 \leq \mathrm{FLI}<60$. Overall, 121,483 persons (0.64\%) developed CRC among 19,048,725 individuals with an FLI $<60$. Mean follow-up time was not significantly different among the three FLI groups $(5.3 \pm 1.1$ years vs. $5.3 \pm$ 1.2 years vs. $5.3 \pm 1.2$ years).

\section{Multivariate analyses for risk factors associated with the development of colorectal cancer}

Risks of developing CRC are shown for each FLI group in Table 2. After controlling for age, BMI, smoking status, alcohol consumption, regular physical exercise, yearly income, and presence of DM, hypertension, and dyslipidemia, participants in $30 \leq \mathrm{FLI}<60$ and FLI $\geq$ 60 groups showed increased risk for CRC (hazard ratios [HRs], 1.07 and 1.13; 95\% confidential intervals [CIs], 1.06 to 1.09 and 1.12 to 1.15 , respectively).

HRs for developing CRC by decile of FLI are depicted in Fig. 2. The HR for developing CRC continuously increased with decile in both men and women.

\section{Influence of BMI on effects of nonalcoholic fatty liver disease on the development of colorectal cancer}

Table 3 shows incidence rates and HRs of CRC by FLI and BMI range. The proportion of participants who took metformin or statin increased depending on BMI range. HRs were calculated after adjusting for age, sex, smoking status, alcohol consumption, regular physical exercise, yearly income, and presence of DM, hypertension, and dyslipidemia. Regardless of BMI range, an FLI $\geq 60$ was significantly correlated with an increased risk of developing CRC. The association of NAFLD with development of $\mathrm{CRC}$ was stronger in individuals with BMI in the range of 18.5 to $23.0 \mathrm{~kg} / \mathrm{m}^{2}$ than in individuals with $\mathrm{BMI}$ in the obese range. Moreover, the association between NAFLD and development of CRC decreased in a dose-dependent manner as BMI increased in obese individuals. HRs of NAFLD for developing CRC for participants with a BMI $<18.5$ and $\geq 18.5 \mathrm{~kg} / \mathrm{m}^{2}$ and $<23 \mathrm{~kg} / \mathrm{m}^{2}$ were 1.44 and 1.29 , respectively, whereas corresponding HRs for BMI of $\geq$ 23 and $<25.0 \mathrm{~kg} / \mathrm{m}^{2}, \geq 25$ and $<30 \mathrm{~kg} / \mathrm{m}^{2}$, and $\geq 30 \mathrm{~kg} / \mathrm{m}^{2}$ were 1.15, 1.11, and 1.09, respectively. 
Table 3. Impact of individual components of the FLI on the risk for developing colorectal cancer by multivariable analyses

\begin{tabular}{|c|c|c|c|c|c|c|c|}
\hline \multirow{2}{*}{$\mathrm{BMI}, \mathrm{kg} / \mathrm{m}^{2}$} & \multicolumn{2}{|c|}{ Usage of medication } & \multirow{2}{*}{ FLI } & \multirow{2}{*}{ Number } & \multirow{2}{*}{ Event } & \multirow{2}{*}{$\mathrm{IR}^{\mathrm{a}}$} & \multirow{2}{*}{$\operatorname{HR}(95 \% \mathrm{CI})^{\mathrm{b}}$} \\
\hline & Metformin & Statin & & & & & \\
\hline \multirow[t]{2}{*}{$\mathrm{BMI}<18.5$} & $13,503(1.5)$ & $24,460(2.8)$ & $<60$ & 884,474 & 3,902 & 0.85 & $0.93(0.90-0.96)$ \\
\hline & & & $\geq 60$ & 1,152 & 16 & 2.94 & $1.44(0.88-2.35)$ \\
\hline \multirow[t]{2}{*}{$18.5 \leq \mathrm{BMI}<23.0$} & $255,440(3.0)$ & $580,027(6.7)$ & $<60$ & $8,596,185$ & 48,673 & 1.06 & 1 (reference) \\
\hline & & & $\geq 60$ & 76,941 & 865 & 2.21 & $1.29(1.21-1.38)$ \\
\hline \multirow[t]{2}{*}{$23.0 \leq \mathrm{BMI}<25.0$} & $259,319(5.0)$ & $621,947(11.9)$ & $<60$ & $4,937,650$ & 34,743 & 1.31 & $1.02(1.00-1.03)$ \\
\hline & & & $\geq 60$ & 281,355 & 2,537 & 1.72 & $1.15(1.10-1.19)$ \\
\hline \multirow[t]{2}{*}{$25.0 \leq \mathrm{BMI}<30.0$} & $401,905(6.7)$ & $953,578(15.8)$ & $<60$ & $4,443,245$ & 33,001 & 1.39 & $1.03(1.02-1.05)$ \\
\hline & & & $\geq 60$ & $1,585,724$ & 12,522 & 1.49 & $1.11(1.08-1.13)$ \\
\hline \multirow[t]{2}{*}{$\mathrm{BMI} \geq 30.0$} & $72,571(9.2)$ & $150,371(19.1)$ & $<60$ & 187,171 & 1,164 & 1.18 & $1.04(0.98-1.10)$ \\
\hline & & & $\geq 60$ & 598,477 & 3,845 & 1.23 & $1.09(1.05-1.13)$ \\
\hline
\end{tabular}

Values are presented as number (\%).

FLI, fatty liver index; BMI, body mass index; IR, incidence rate; HR, hazard ratio; CI, confidence interval.

${ }_{1,000}$ person-year.

${ }^{\mathrm{b}}$ Data are adjusted by age, smoking status, alcohol consumption, regular physical exercise, and yearly income.

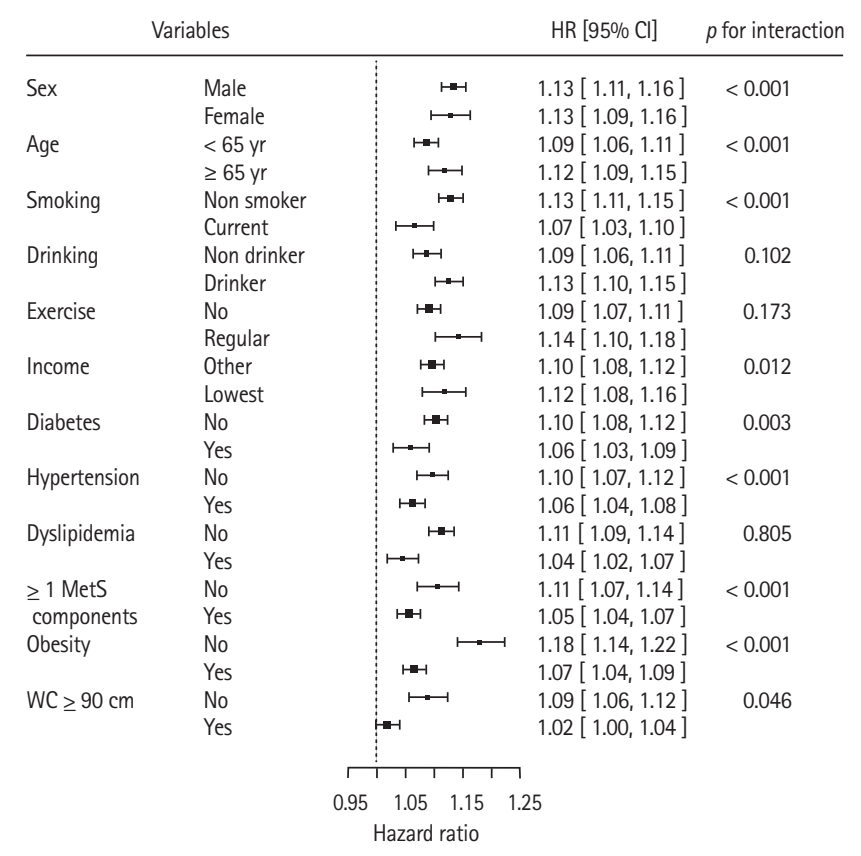

Figure 3. Forest plot of hazard ratios by subgroups according to variables. Multivariate logistic regression analysis was used after controlling for age, smoking status, alcohol consumption, regular exercise, and yearly income. HR, hazard ratio; CI, confidence interval; MetS, metabolic syndrome; WC, waist circumference.

\section{Subgroup analyses according to age, smoking status, alcohol consumption, and metabolic health status}

Subgroup analyses were performed according to sex, age, smoking status, alcohol consumption, regular physical exercise, yearly income, and the presence of DM, hypertension, and dyslipidemia obesity, and a combination of hypertension and dyslipidemia. Fig. 3 shows HRs of CRC and $p$ values for interaction by subgroup after controlling for age, sex, smoking status, alcohol consumption, regular physical exercise, and yearly income.

For NAFLD defined by FLI $\geq 60$, participants aged 65 years or older showed a higher risk for CRC than the other age groups (HR, 1.12; 95\% CI, 1.09 to 1.15 vs. HR, 1.09; 95\% CI, 1.06 to 1.11; $p$ for interaction < 0.001) (Fig. 3). Nonsmokers or ex-smokers, alcohol consumers (<30 $\mathrm{g}$ (day), and persons with the lowest quintile of yearly income showed greater association with development CRC. Moreover, associations between NAFLD and the development of CRC were more prominent in the absence of DM, hypertension, obesity and abdominal obesity (for all $p$ for interactions $<0.05$ ).

Non-diabetic, normotensive, and non-dyslipidemic individuals with NAFLD had a higher risk for developing CRC than persons who had one or more elements of metabolic syndrome (DM, hypertension, or dyslipid- 
emia) (HR, 1.11; 95\% CI, 1.07 to 1.14 vs. HR, 1.05; 95\% CI, 1.04 to 1.07 ; $p$ for interaction $<0.001$ ).

\section{Effect of changes in fatty liver index score on developing colorectal cancer}

Among the study population, 6,739,987 persons had undergone consecutive biennial evaluations. To evaluate the effect of changes in FLI on developing CRC, these participants were subclassified to four groups by changes in FLI over a 2-year follow-up interval (Supplementary Table 2).

Individuals whose FLI had been less than 60 at baseline but became 60 or greater at follow-up showed an increased HR for CRC than individuals whose FLI remained less than 60 (HR, 1.09; 95\% CI, 1.04 to 1.15). Persons whose FLI $\geq 60$ at baseline consistently showed an increased risk for development of CRC regardless of FLI change at follow-up (Supplementary Table 2).

\section{DISCUSSION}

In the present nationwide study, which involved a large cohort of Korean individuals, NAFLD as defined by the FLI $\geq 60$ was correlated with an increase up to $13 \%$ for development of CRC. This association was dose-dependent and more prominent in persons with a normal BMI compared to obese persons after controlling for age, sex, smoking status, alcohol consumption, regular physical exercise, and yearly income. Moreover, normoglycemic, normotensive, and non-dyslipidemic individuals with NAFLD had a higher risk for developing CRC compared with persons who had one or more elements of metabolic syndrome (DM, hypertension, or dyslipidemia).

Several studies have evaluated the relationship between NAFLD and CRC [11,12,22]. Wong et al. [12] showed that patients with NAFLD had a higher prevalence of colorectal adenomas and CRC than their counterparts. In a large retrospective cohort study of 5,517 Korean women, a significant relationship between NAFLD and colorectal neoplasm was observed [11], consistent with the current finding. However, the association between NAFLD and CRC is not consistently reported. Touzin et al. [22] reported no significant difference in the development of neoplasm, despite the higher mean number of adenomas in patients with NAFLD than in the control group. Because benign colonic polyps were frequently missing in the NHIC claim data, the relationship between NAFLD and colonic adenoma was not addressed in the present study.

Within the Korean cohort, mean age at diagnosis of NAFLD was $41.6 \pm 8.7$, while the incidence of CRC rapidly increases after age 50 and peaks at ages 65 to 69 $[2,23]$. This discrepancy in ages at diagnosis can be a confounding factor; that is, the incidence of CRC was highest for individuals with $30 \leq \mathrm{FLI}<6$ o before adjustment of age. However, after controlling age, HR of CRC among participants with FLI $\geq 60$ was higher than HR among individuals with $30 \leq$ FLI $<60$ (Table 2). These results remained consistent after controlling several covariates other than age.

Although the proposed mechanism for how NAFLD affects carcinogenesis is not completely understood, NAFLD may represent a condition of insulin resistance and a proinflammatory state [24]. Insulin and insulin-like growth factors may promote development of colorectal neoplasm through their proliferative and antiapoptotic effects. Moreover, patients with NAFLD have reduced expression of adiponectin, which has anti-inflammatory properties $[25,26]$.

Given that obesity is the usual cause for the development of NAFLD, a condition linked to CRC [18], Lower HR for NAFLD and development of CRC in obese individuals or participants with a waist circumference > $90 \mathrm{~cm}$ is an interesting finding. Non-obese NAFLD participants could represent a different disease entity with prominent visceral fat distribution and special genetic predisposition when compared to obese individuals with NAFLD [27]. Visceral fat is a source of free fatty acids and pro-inflammatory cytokines [27]. High fructose diets are reported to play an important role in the development of NAFLD, particularly in young and nonobese populations [28]. Fructose is proinflammatory, causing intracellular adenosine triphosphate depletion, uric acid production and subsequent cellular injury [29]. These actions might be related to CRC carcinogenesis. By contrast, the higher HR of NAFLD and CRC in participants without diabetes, hypertension, and dyslipidemia than in individuals who have at least one of the three elements of metabolic syndrome might be a result of anti-cancer properties of a statin [30] or metformin 
[31]. The proportion of individuals who were administered metformin or statin increased as BMI increased (Table 3).

However, more prominent associations between NAFLD and development of CRC in individuals with normal BMI than obese individuals should not be interpreted as a healthy obesity theory. Rather, this study suggests the important insight that NAFLD (or the high FLI) may be a more sensitive early marker for development of CRC than BMI or metabolic syndrome criteria. Rapidly increasing incidence of CRC and previously undocumented high incidence among young adults indicates that CRC screening in this group may become an important issue [32].

Changes in FLI score for individuals who participated in consecutive biennial exams indicates an increased risk of CRC in participants who became FLI-based NAFLD at the 2-year follow-up. However, established FLIbased NAFLD patients whose FLI went down to less than 60 during the follow-up period did not show reduced risk. Participants in the $30 \leq$ FLI $<60$ may have a condition that encourages development of CRC, or some confounding factors may reverse any beneficial effect of a reduction in FLI score. Whether reduction in FLI score could lead to a decreased risk for CRC needs further long-term, well-organized studies.

The limitations of the present study include lack of data from imaging modalities and biopsy to evaluate NAFLD, and some misclassification may exist. Previous external validation data from Taiwan shows that FLI can accurately identify ultrasonographic fatty liver in a large-scale population [33]. The study suggests that optimal cut-off values are lower than cut-offs for Western populations; FLI $\geq 35$ and $\geq 20$ to rule in ultrasonographic fatty liver for men and women. Indeed, the risk for CRC increased in participants with an FLI $\geq 30$, suggesting that risk stratification using FLI can be useful. To compensate for this limitation, a sensitivity analysis used a simple AST/ALT ratio. An increasing AST/ALT ratio is associated with advanced fibrosis [34]. When 1.0 was used as a cut-off value, an AST/ALT ratio $\geq 1$ was consistently associated with an increased risk for CRC in multivariable analyses (Supplementary Table 3).

A lack of information on histological type and stage is also a limitation. Rectal cancer could not be differentiated from CRC because most claims did not precisely specify tumor location. Although the association was significant, it was modest.

Nonetheless, the largest nationwide database ever used formed the basis for the study. The sample size of this study exceeds the sum of previous case-control or cohort studies. This study presents preliminary data that should promote further studies on individuals with high FLI.

NAFLD defined by the FLI is significantly associated with the presence of CRC in asymptomatic patients undergoing screening. This finding provides a clinical means for risk stratification of CRC and for screening strategies using FLI.

\section{KEY MESSAGE}

1. High fatty liver index score is correlated with development of colorectal cancer.

2. High fatty liver index is correlated with colorectal cancer regardless of body mass index, but the association was more prominent in persons with a normal body mass index.

3. Individuals with a fatty liver index $\geq 60$ require close consideration for development of colorectal cancer even in the absence of obesity or other elements of metabolic syndrome.

\section{Conflict of interest}

No potential conflict of interest relevant to this article was reported.

\section{Acknowledgments}

This work was supported by the Korea Institute of Planning and Evaluation for Technology in Food, Agriculture, Forestry and Fisheries (IPET) through the High Value-added Food Technology Development Program, and funded by the Ministry of Agriculture, Food and Rural Affairs (MAFRA) (No. 116017032HDo3o).

\section{REFERENCES}

1. Jemal A, Bray F, Center MM, Ferlay J, Ward E, Forman D. Global cancer statistics. CA Cancer J Clin 2011;61:69-90.

2. Haggar FA, Boushey RP. Colorectal cancer epidemiology: 
incidence, mortality, survival, and risk factors. Clin Colon Rectal Surg 2009;22:191-197.

3. Shin A, Kim KZ, Jung KW, et al. Increasing trend of colorectal cancer incidence in Korea, 1999-2009. Cancer Res Treat 2012;44:219-226.

4. Ferlay J, Soerjomataram I, Dikshit R, et al. Cancer incidence and mortality worldwide: sources, methods and major patterns in GLOBOCAN 2012. Int J Cancer 2015;136:Е359-E386.

5. Alberti KG, Eckel RH, Grundy SM, et al. Harmonizing the metabolic syndrome: a joint interim statement of the International Diabetes Federation Task Force on Epidemiology and Prevention; National Heart, Lung, and Blood Institute; American Heart Association; World Heart Federation; International Atherosclerosis Society; and International Association for the Study of Obesity. Circulation 2009;120:1640-1645.

6. Esposito K, Chiodini P, Colao A, Lenzi A, Giugliano D. Metabolic syndrome and risk of cancer: a systematic review and meta-analysis. Diabetes Care 2012;35:2402-2411.

7. McMillan DC, Sattar N, McArdle CS. ABC of obesity. Obesity and cancer. BMJ 2006;333:1109-1111.

8. Charlton MR, Burns JM, Pedersen RA, Watt KD, Heimbach JK, Dierkhising RA. Frequency and outcomes of liver transplantation for nonalcoholic steatohepatitis in the United States. Gastroenterology 2011;141:1249-1253.

9. Yki-Jarvinen $\mathrm{H}$. Non-alcoholic fatty liver disease as a cause and a consequence of metabolic syndrome. Lancet Diabetes Endocrinol 2014;2:901-910.

10. Lonardo A, Ballestri S, Marchesini G, Angulo P, Loria P. Nonalcoholic fatty liver disease: a precursor of the metabolic syndrome. Dig Liver Dis 2015;47:181-190.

11. Lee YI, Lim YS, Park HS. Colorectal neoplasms in relation to non-alcoholic fatty liver disease in Korean women: a retrospective cohort study. J Gastroenterol Hepatol 2012;27:91-95.

12. Wong VW, Wong GL, Tsang SW, et al. High prevalence of colorectal neoplasm in patients with non-alcoholic steatohepatitis. Gut 2011;60:829-836.

13. Bedogni G, Bellentani S, Miglioli L, et al. The fatty liver index: a simple and accurate predictor of hepatic steatosis in the general population. BMC Gastroenterol 2006;6:33.

14. Kozakova M, Palombo C, Eng MP, et al. Fatty liver index, gamma-glutamyltransferase, and early carotid plaques. Hepatology 2012;55:1406-1415.
15. Calori G, Lattuada G, Ragogna F, et al. Fatty liver index and mortality: the Cremona study in the 15th year of follow-up. Hepatology 2011;54:145-152.

16. Lee YH, Kim JE, Roh YH, et al. The combination of vitamin $\mathrm{D}$ deficiency and mild to moderate chronic kidney disease is associated with low bone mineral density and deteriorated femoral microarchitecture: results from the KNHANES 2008-2011. J Clin Endocrinol Metab 2014;99:3879-3888.

17. Oh SW. Obesity and metabolic syndrome in Korea. Diabetes Metab J 2011;35:561-566.

18. Tilg H, Diehl AM. NAFLD and extrahepatic cancers: have a look at the colon. Gut 2011;60:745-746.

19. Lee YH, Jung KS, Kim SU, et al. Sarcopaenia is associated with NAFLD independently of obesity and insulin resistance: nationwide surveys (KNHANES 2008-2011). J Hepatol 2015;63:486-493.

20. Lee JH, Kim D, Kim HJ, et al. Hepatic steatosis index: a simple screening tool reflecting nonalcoholic fatty liver disease. Dig Liver Dis 2010;42:503-508.

21. Gastaldelli A, Kozakova M, Hojlund K, et al. Fatty liver is associated with insulin resistance, risk of coronary heart disease, and early atherosclerosis in a large European population. Hepatology 2009;49:1537-1544.

22. Touzin NT, Bush KN, Williams CD, Harrison SA. Prevalence of colonic adenomas in patients with nonalcoholic fatty liver disease. Therap Adv Gastroenterol 2011;4:169176.

23. Jeong EH, Jun DW, Cho YK, et al. Regional prevalence of non-alcoholic fatty liver disease in Seoul and Gyeonggi-do, Korea. Clin Mol Hepatol 2013;19:266-272.

24. Wong VW, Hui AY, Tsang SW, et al. Metabolic and adipokine profile of Chinese patients with nonalcoholic fatty liver disease. Clin Gastroenterol Hepatol 2006;4:1154-1161.

25. Wong VW, Wong GL, Tsang SW, et al. Genetic polymorphisms of adiponectin and tumor necrosis factor-alpha and nonalcoholic fatty liver disease in Chinese people. $J$ Gastroenterol Hepatol 2008;23:914-921.

26. Hui JM, Hodge A, Farrell GC, Kench JG, Kriketos A, George J. Beyond insulin resistance in NASH: TNF-alpha or adiponectin? Hepatology 2004;40:46-54.

27. Yousef MH, Al Juboori A, Albarrak AA, Ibdah JA, Tahan V. Fatty liver without a large "belly": magnified review of non-alcoholic fatty liver disease in non-obese patients. World J Gastrointest Pathophysiol 2017;8:100-107.

28. Zelber-Sagi S, Nitzan-Kaluski D, Goldsmith R, et al. Long 
term nutritional intake and the risk for non-alcoholic fatty liver disease (NAFLD): a population based study. J Hepatol 2007;47:711-717.

29. Basaranoglu M, Basaranoglu G, Bugianesi E. Carbohydrate intake and nonalcoholic fatty liver disease: fructose as a weapon of mass destruction. Hepatobiliary Surg Nutr 2015;4:109-116.

30. Poynter JN, Gruber SB, Higgins PD, et al. Statins and the risk of colorectal cancer. N Engl J Med 2005;352:2184-2192.

31. Zhang ZJ, Zheng ZJ, Kan H, et al. Reduced risk of colorectal cancer with metformin therapy in patients with type 2 diabetes: a meta-analysis. Diabetes Care 2011;34:2323-2328.
32. Sung JJ, Ng SC, Chan FK, et al. An updated Asia Pacific Consensus Recommendations on colorectal cancer screening. Gut 2015;64:121-132.

33. Yang BL, Wu WC, Fang KC, et al. External validation of fatty liver index for identifying ultrasonographic fatty liver in a large-scale cross-sectional study in Taiwan. PLoS One 2015;10:e0120443.

34. McPherson S, Stewart SF, Henderson E, Burt AD, Day CP. Simple non-invasive fibrosis scoring systems can reliably exclude advanced fibrosis in patients with non-alcoholic fatty liver disease. Gut 2010;59:1265-1269. 


\section{Supplementary Table 1. Demographics of the study enrolees}

\begin{tabular}{|c|c|c|c|}
\hline Variable & FLI $<60(n=19,048,725)$ & $\mathrm{FLI} \geq 60(\mathrm{n}=2,543,649)$ & $p$ value \\
\hline Male sex & $8,489,867(44 \cdot 57)$ & $1,999,161(78.59)$ & $<0.001$ \\
\hline Age, yr & $47 \cdot 6 \pm 14 \cdot 5$ & $48.5 \pm 12.9$ & $<0.001$ \\
\hline$<40$ & $5,612,971(29.47)$ & $667,250(26.23)$ & $<0.001$ \\
\hline $40-60$ & $10,733,947(56.35)$ & $1,555,383(61.15)$ & $<0.001$ \\
\hline$\geq 60$ & $2,701,807(14.18)$ & $321,016(12.62)$ & $<0.001$ \\
\hline Current smoker & $3,903,300(20.49)$ & $1,001,952(39 \cdot 39)$ & $<0.001$ \\
\hline Alcohol consumption ${ }^{\mathrm{a}}$ & $7,862,705(41.28)$ & $1,537,409(60.44)$ & $<0.001$ \\
\hline Regular exercise $^{\mathrm{b}}$ & $3,379,448(17.74)$ & $426,799(16.78)$ & $<0.001$ \\
\hline Lower quintile of yearly income & 4,063,197 (21.33) & 491,132 (19.31) & $<0.001$ \\
\hline BMI, $\mathrm{kg} / \mathrm{m}^{2}$ & $23.1 \pm 2.8$ & $28.1 \pm 3.1$ & $<0.001$ \\
\hline $\mathrm{BMI}, \geq 25 \mathrm{~kg} / \mathrm{m}^{2}$ & $4,630,416(24 \cdot 31)$ & $2,184,201(85.87)$ & $<0.001$ \\
\hline WC, cm & $78.1 \pm 8.1$ & $92.4 \pm 6.9$ & $<0.001$ \\
\hline $\mathrm{WC} \geq 90 \mathrm{~cm}$ & $4,274,102(22.44)$ & $1,821,347(71.6)$ & $<0.001$ \\
\hline $\mathrm{SBP}, \mathrm{mmHg}$ & $120.9 \pm 14.9$ & $130 \pm 14.6$ & $<0.001$ \\
\hline $\mathrm{DBP}, \mathrm{mmHg}$ & $75.1 \pm 9.8$ & $81.4 \pm 9.9$ & $<0.001$ \\
\hline Serum glucose, mg/dL & $95 \cdot 7 \pm 21$ & $108.2 \pm 32.3$ & $<0.001$ \\
\hline Serum cholesterol, mg/dL & $192.6 \pm 35 \cdot 9$ & $211.3 \pm 39.5$ & $<0.001$ \\
\hline Serum triglyceride, $\mathrm{mg} / \mathrm{dL}^{\mathrm{c}}$ & $100.18(100.16-100.21)$ & $226.41(226.27-226.55)$ & $<0.001$ \\
\hline $\mathrm{ALT}, \mathrm{IU} / \mathrm{L}^{\mathrm{c}}$ & $20.01(20.01-20.02)$ & $37.71(37.69-37.74)$ & $<0.001$ \\
\hline $\mathrm{AST}, \mathrm{IU} / \mathrm{L}^{\mathrm{c}}$ & $23.08(23.07-23.08)$ & $31.39(31.37-31.4)$ & $<0.001$ \\
\hline GGT, IU/L $\mathrm{L}^{\mathrm{c}}$ & $22.69(22.68-22.7)$ & $64.45(64.39-64.5)$ & $<0.001$ \\
\hline Hypertension & $4,394,122(23.07)$ & $1,169,468(45.98)$ & $<0.001$ \\
\hline Dyslipidaemia $^{\mathrm{d}}$ & $3,248,048(17.05)$ & $920,307(36.18)$ & $<0.001$ \\
\hline Diabetes & $1,439,820(7 \cdot 56)$ & $505,886(19.89)$ & $<0.001$ \\
\hline$\geq 1$ of metabolic syndrome component ${ }^{\mathrm{e}}$ & $6,586,701(34.58)$ & $1,666,824(65 \cdot 53)$ & $<0.001$ \\
\hline Developing CRC & $121,483(0.64)$ & $19,785(0.78)$ & $<0.001$ \\
\hline Follow-up duration, yr & $5 \cdot 3 \pm 1.1$ & $5 \cdot 3 \pm 1.2$ & $<0.001$ \\
\hline
\end{tabular}

Values are presented as number (\%) or mean \pm SD.

FLI, fatty liver index; BMI, body mass index; WC, waist circumference; SBP, systolic blood pressure; DBP, diastolic blood pressure; ALT, alanine transaminase; AST, aspartate aminotransferase; GGT, gamma glutamyltransferase; CRC, colorectal cancer.

${ }^{a}$ Persons who alcohol consumption $\geq 30 \mathrm{~g} /$ day were initially excluded.

${ }^{b}$ Persons who did not perform high intensity of activity $\geq 3 /$ week or moderate intensity of activity $\geq 5 /$ week.

${ }^{\mathrm{c}}$ Geometric mean (95\% confidence interval).

${ }^{\mathrm{d}}$ Triglyceride $\geq 150 \mathrm{mg} / \mathrm{dL}$ or user of lipid lowering drug.

${ }^{\mathrm{e}}$ Having more than 1 of component among hypertension, dyslipidaemia and diabetes mellitus. 
Choi YJ, et al. NAFLD and colorectal cancer screening

Supplementary Table 2. Incidence and hazard ratios of colorectal cancer according to change of FLI at 2 years follow-up compared to the baseline

\begin{tabular}{|c|c|c|c|c|c|c|c|c|}
\hline \multicolumn{2}{|c|}{ FLI } & \multirow{2}{*}{ Number } & \multirow{2}{*}{$\mathrm{CRC}, \mathrm{n}$} & \multirow{2}{*}{$\begin{array}{c}\text { Duration, } \\
\text { person-years }\end{array}$} & \multirow{2}{*}{$\mathrm{IR}^{\mathrm{a}}$} & \multicolumn{3}{|c|}{$\mathrm{HR}(95 \% \mathrm{CI})$} \\
\hline Pre & Post & & & & & Model $1^{b}$ & Model $2^{c}$ & Model $3^{\mathrm{d}}$ \\
\hline \multirow[t]{2}{*}{$<60$} & $<60$ & $5,707,716$ & 28,811 & $24,139,856$ & 1.19 & 1 (reference) & 1 (reference) & 1 (reference) \\
\hline & $\geq 60$ & 293,991 & 1,588 & $1,227,701$ & 1.29 & $1.09(1.03-1.14)$ & $1.10(1.04-1.15)$ & $1.09(1.04-1.15)$ \\
\hline \multirow[t]{2}{*}{$\geq 60$} & $<60$ & 263,706 & 2,053 & $1,103,286$ & 1.86 & $1.56(1.49-1.63)$ & $1.29(1.23-1.35)$ & $1.28(1.22-1.34)$ \\
\hline & $\geq 60$ & 474,574 & 2,647 & $1,984,898$ & 1.33 & $1.12(1.08-1.16)$ & $1.14(1.09-1.19)$ & $1.13(1.08-1.17)$ \\
\hline
\end{tabular}

FLI, fatty liver index; CRC, colorectal cancer; IR, incidence rate; HR, hazard ratios; CI, confidential interval.

${ }^{\mathrm{a}} 1,000$ person-year.

${ }^{\mathrm{b}}$ Unadjusted.

${ }^{\mathrm{c}}$ Data are adjusted for age, sex.

${ }^{\mathrm{d}}$ Data are adjusted for age, sex, smoking status, alcohol consumption, regular physical exercise and income. 
Supplementary Table 3. Results of multivariable analyses of the impact of the AST/ALT ratio on the risk of colorectal cancer in the Korean population

\begin{tabular}{|c|c|c|c|c|c|c|c|}
\hline \multirow{2}{*}{ AST/ALT } & \multirow{2}{*}{ Number } & \multirow{2}{*}{ Event, $\mathrm{n}$} & \multirow{2}{*}{$\begin{array}{c}\text { Duration, } \\
\text { person-years }\end{array}$} & \multirow{2}{*}{$\mathrm{IR}^{\mathrm{a}}$} & \multicolumn{3}{|c|}{$\operatorname{HR}(95 \% \mathrm{CI})$} \\
\hline & & & & & Model $1^{b}$ & Model $2^{c}$ & Model $3^{\mathrm{d}}$ \\
\hline$<1$ & $7,247,646$ & 43,567 & $38,854,776$ & 1.12 & 1 (reference) & 1 (reference) & 1 (reference) \\
\hline $1 \leq$ & $14,344,728$ & 97,701 & $76,143,966$ & 1.28 & $1.02(1.01-1.03)$ & $1.04(1.02-1.05)$ & $1.05(1.04-1.06)$ \\
\hline
\end{tabular}

AST, aspartate aminotransferase; ALT, alanine aminotransferase; IR, incidence rate; HR, hazard ratios; CI, confidential interval. ${ }_{1,000}$ person-year.

${ }^{\mathrm{b}}$ Data are adjusted for age, sex.

${ }^{\mathrm{c}}$ Data are adjusted for age, sex, smoking status, alcohol consumption, regular physical exercise and income.

${ }^{\mathrm{d}}$ Data are adjusted for age, sex, smoking status, alcohol consumption, regular physical exercise, income, diabetes mellitus, hypertension, and dyslipidemia. 